\title{
Characteristics of a Wireless Three-hop Relay Channel with the Presence of Rayleigh Fading
}

\author{
Dragana Krstic ${ }^{1}$, Petar Nikolic ${ }^{2}$, Zoran Popovic ${ }^{3}$, and Mihajlo Stefanovic ${ }^{1}$ \\ ${ }^{1}$ Faculty of Electronic Engineering, University of Nǐ̌, Nǐ̌s, Serbia \\ 2 TigarTyres, Pirot, Serbia \\ ${ }^{3}$ Higher Technical School of Professional Studies, Zvečan, Serbia
}

https://doi.org/10.26636/jtit.2020.144020

\begin{abstract}
In this paper, a wireless three-hop relay communication system operating in a Rayleigh multipath fading environment is analyzed. The output signal from such a system is a product of signal envelopes from all sections, meaning that it is actually a product of three random variables (RVs) with Rayleigh distribution. We considered here the first-order characteristics: probability density function (PDF), cumulative distribution function and outage probability (OP). Then, the moments and amount of fading (AoF) were derived in the closed forms. The second order characteristics we present include the following: level crossing rate (LCR) and average fade duration (AFD). A few graphs are given to show the impact of the specific parameters of the wireless three-hop relay system.
\end{abstract}

Keywords-first order performance, Rayleigh fading, second order performance, three-hop relay telecommunication systems.

\section{Introduction}

The output signal of a wireless relay system is a product of signal envelopes from each section. Due to this fact, the performance of products of a higher number of random variables (RVs) has become an important topic over the past decade. RV products are applied in wireless channel modeling, multi-hop relay systems, multiple input multiple output (MIMO) keyhole systems, cascaded channels with fading, but also in other natural sciences, such as biology and physics (especially quantum physics), and also in social sciences, namely econometrics [1]-[3].

The first studies in this field were carried out in the previous century [4]. Donahue et al. were the first to study distributions of the products of two RVs [5]-[8]. In the new millennium, research in that field commenced again [9]-[12], and newer papers greatly impact practical applications in wireless relay communication systems [13]-[16].

Previously published papers focused primarily on products of two RVs. By using the level crossing rate (LCR) of a product of two Nakagami- $m$ RVs from [3], the aver- age fade duration (AFD) of a wireless relay system which works in a Nakagami- $m$ fading channel is obtained. Further, probability density function (PDF) of the product of Rayleigh, exponentially, Nakagami- $m$ and gamma RVs is calculated based on the Mellin transform in [17]. The authors in [18] present double fading channels with a lineof-sight (LOS) channel (double Rician fading), typical of keyhole MIMO models.

An analysis of the features of the product of two independent $\kappa-\mu$ distributed RVs is given in [19]. The analytical formulas for PDF and cumulative distribution function (CDF) are derived. In [20], new formulas for PDF, CDF, and moment generating function (MGF) are given, based on their expressions for higher order moments, amount of fading (AoF) and the channel quality estimation index in closed-forms. The formulas for outage probability (OP), average channel capacity $(\mathrm{aCC})$, average symbol error probability (aSEP) and average bit error probability (aBEP) are derived as well. Some properties of the product of independent RVs with a general $\alpha-\mu$ distribution, such as: PDF, CDF, moments, OP and AoF, are presented in [13] in closed-form expressions. This type of fading impacts also the dual-hop wireless communication system with a nonregenerative relay that is considered in [21]. The authors derived closed-form expressions for MGF, CDF, and PDF of the harmonic mean of the end-to-end signal-to-noise ratio (SNR), using the $\alpha-\mu$ fading model. They also determined closed-form expressions for the end-to-end capacity and outage capacity of that system.

Dual-hop communication systems are known as tools enabling to extend coverage and reduce transmit power in scenarios where one-hop communication is not possible. An example of a dual-hop communication system is presented in Fig. 1 and in many papers such as [21]-[23]. PDF, CDF and MGF of the total received SNR of a dual-hop amplifyand-forward relay (AFR) transmission over non-identical Rician fading channels are obtained in the form of rapidly 
converging infinite sums in [23]. Then, OP and aBER are calculated.

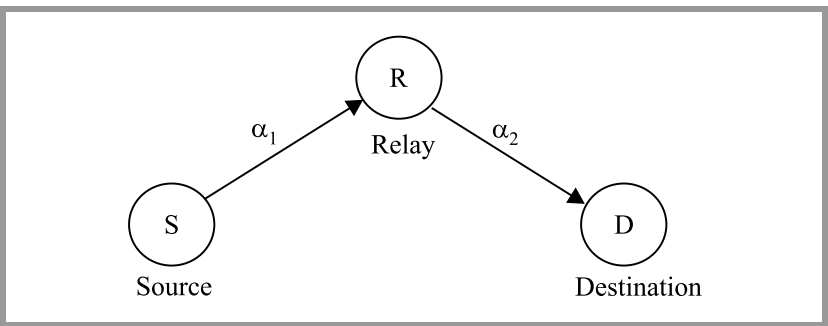

Fig. 1. Example of a dual-hop wireless communication system [22].

One group of authors has observed in [24] such a scenario for general $\alpha-\eta-\mu$ fading distribution, performed an analysis of SNR for dual-hop amplify-and-forward (AF) cooperative communication systems, and derived ergodic capacity. The first-order outage statistics (CDF, OP and aBER) of an asymmetrical radio-frequency (RF) optical wireless (OW) dual-hop AFR communication system have been processed in [25]. Their system is modeled as a product of independent Nakagami- $m$ and double squared Nakagami- $m$ (or gamma-gamma) random processes. A similar scenario was also analyzed in [26].

By dint of spatial diversity and dual-hop relaying widely adopted in cooperative wireless systems, communication coverage may be effectively extended and the potentially severe channel fading may be mitigated [27]. The AF relaying protocol has been applied, since it generates an effective tradeoff between low implementation costs and a sufficient performance level. So, satellite communication represents a direct application of dual-hop AF relaying configurations. New calculations for measuring important performance parameters of a satellite relay have been performed in the presence of shadowed Rician fading for OP, ASEP and ergodic capacity of the end-to-end SNR.

One of the newest papers focusing on this area is [28]. Its authors analyzed the performance of a dual-hop cooperative decode-and-forward (DF) relaying system with beamforming relying on different adaptive transmission techniques over $\kappa-\mu$ shadowed fading channels.

Although three-hop relay systems have been discussed before [29]-[31], our group of authors is still among the first dealing with this telecommunication system configuration. So, we started observing the products of three RVs with different distributions and deriving system performance in closed forms [32]-[36]. The product of three $\mathrm{RVs}$ in relay telecommunication channels when multipath fading is present, is given in [32]. The LCR of the product of Nakagami- $m$ RV, Rician RV and Rayleigh RV is obtained in a closed form in [33]. The product of three Nakagami$m \mathrm{RVs}$ is analyzed in [34] and [35], and the statistics of the second order of the product of three Rician random variables is analyzed in [36].

Before our investigations were conducted, a survey on packet retrieval for a three-hop routing protocol in a hybrid wireless network was presented in [29]. On the other hand, paper [30] presents a distributed three-hop routing protocol (DTR) for hybrid wireless networks, combining the advantages of mobile ad-hoc networks and infrastructure wireless networks, and achieving ultra-high performance.

Fixed cellular relays used in modern wireless communication systems are powerful technique allowing to improve reliability and to increase coverage under shadowing propagation conditions and limited transmit power conditions [31]. There, the lower and upper bounds of the sum capacity of different relaying strategies are analyzed. Figure 2 presents an example of a spectral efficiency sharing model deployed in two adjacent base stations, as described in this article [Fig. 1, 31]. In Fig. 3, a three-hop relay with two channels is shown [37].

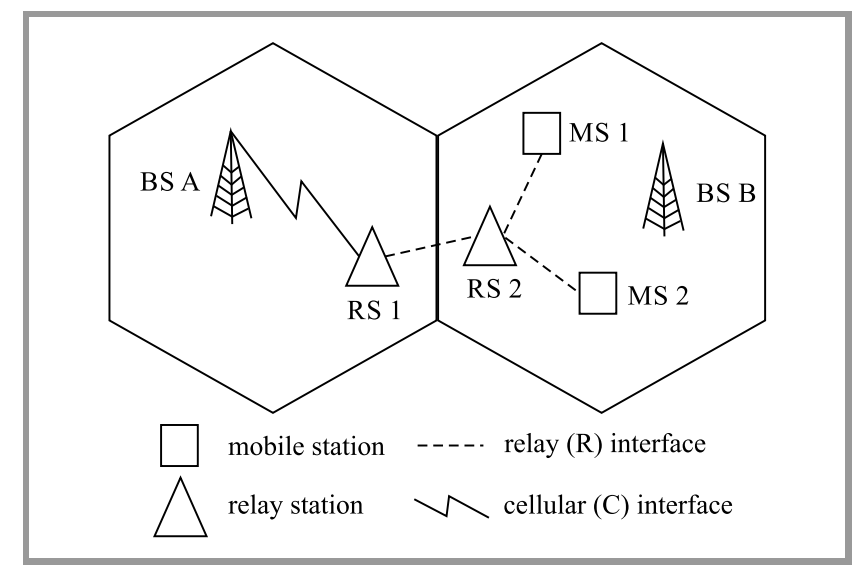

Fig. 2. Example of a dual-hop communication system [31].

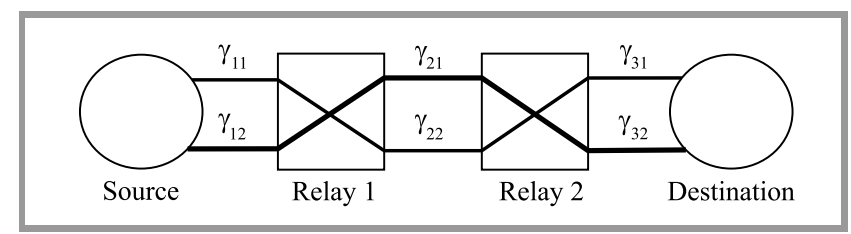

Fig. 3. A three-hop relay with two channels [37].

In a mobile ad-hoc network, data is routed to its destination through the intermediate nodes in a multi-hop manner. One of multi-hop system models, using antenna selection per hop, is plotted in Fig. 4 [38].

In the aforementioned work, OP of multi-hop MIMO relaying with a transmit antenna selection and an ideal relay gain over the Rayleigh fading environment is observed.

Further, in [39]-[41], multiple RVs with different distributions are studied. So, PDF of the product of $n$-Rayleigh distribution is obtained in [39]. Distribution is derived by dint of inverse Mellin transform, and is presented using the Meijer G-function. An analysis of the second order statistics of the AF multi-hop Rayleigh fading environment is presented in [40]. The output signal from this cascaded channel is a product of N Rayleigh fading signal envelopes, written as $\mathrm{N}^{*}$ Rayleigh RV. The formulas for LCR and AFD are derived for such an RV. Then, PDF, CDF, and mo- 


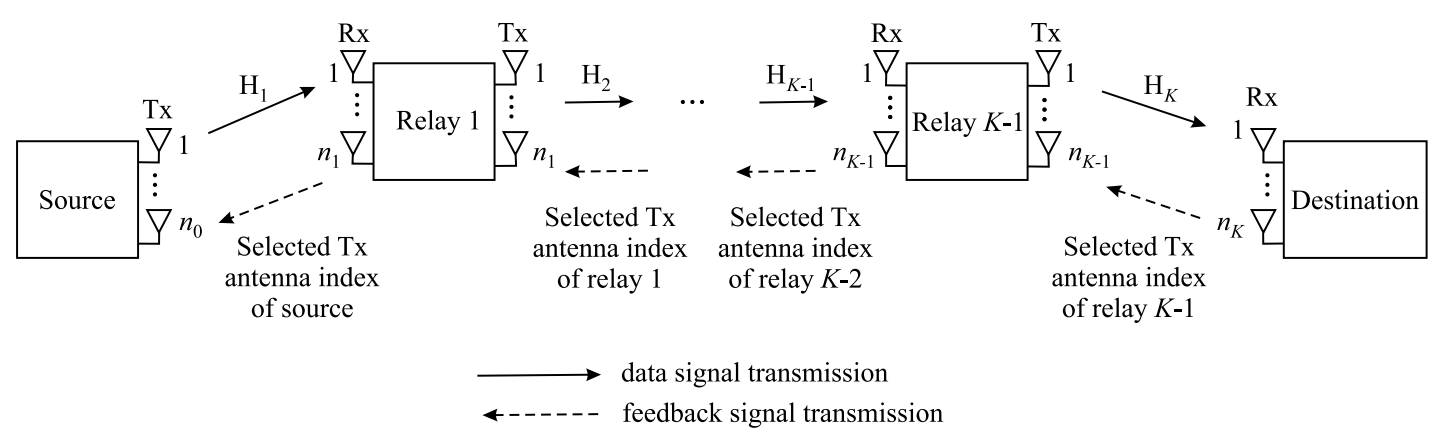

Fig. 4. A multi-hop system using antenna selection per hop [38].

ments of the $\mathrm{N}^{*}$ Nakagami distribution are worked out in closed forms across the Meijer's G-function in [41]. The expressions are derived for OP, AoF, and aBEP, for a few modulation formats.

Unlike previously published works, we derived some second-order performance parameters in their closed forms, because time variations of the fading environments are characterized by second order statistics. This applies, in particular, to LCR and AFD of wireless links. These two important statistics are used for Markov modeling of wireless channels [42] and for designing diversity combiners which will be used in wireless communication systems to mitigate the impact of fading. Consequently, they are important topics of many available articles.

This paper is concerned primarily with a three-hop wireless relay system functioning in the presence of Rayleigh fading. The multi-hop relay system's output signal is in fact a product of output signals from all sections (hops) [43]. For such a system, we obtained in [44]: probability density function, cumulative distribution function, outage probability, and the following second order system performance: level crossing rate and average fade duration. To complete the set of first order system performance, moments and amount of fading will be calculated in closed forms in this article.

This paper consists of four sections. In introduction, the topic is presented and the literature is analyzed. In Section 2, first order system characteristics of the product of three RVs are analyzed. Second order characteristics for triple Rayleigh RV are given in Section 3. All performance parameters are validated by graphical expressions. Section 4 presents the conclusion and proposals concerning future work.

\section{First Order Characteristics of the Product of Three Rayleigh Random Variables}

The multipath transmission in wireless systems is shown in Fig. 5 [45]. The path from the transmitter to the receiver consists of more distinct paths. In this figure, three such paths between the transmitter and the receiver are pre- sented. In this scenario, the line-of-sight path does not exist. Only multiple indirect paths are available, and this is a Rayleigh channel.

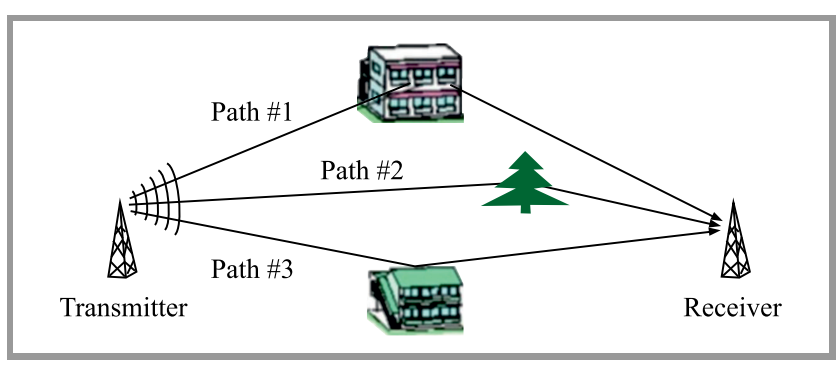

Fig. 5. Multipath transmission in wireless systems without a direct path between the transmitter and the receiver [45].

The signal envelopes represent random variables $x_{i}$, $i=1,2,3$ which are Rayleigh distributed [46]:

$$
p_{x_{i}}\left(x_{i}\right)=\frac{2 x_{i}}{\Omega_{i}} \mathrm{e}^{-\frac{x_{i}^{2}}{\Omega_{i}}}, \quad x_{i} \geq 0,
$$

where $\Omega_{i}=E\left\{x_{i}^{2}(t)\right\}, 1 \leq i \leq 3$, are the mean powers of the $i$-th RV [Eq. (1), 40].

We assume that random variable $x$ is a product of three Rayleigh RVs [Eq. (2), 44]:

$$
x=\prod_{i=1}^{3} x_{i} .
$$

Based on Eq. (2), it is valid that: $x_{1}=\frac{x}{\left(x_{2} x_{3}\right)}$.

\subsection{PDF of the Product of Three Rayleigh RVs}

The probability density function of the product of three Rayleigh RVs $x$ is given by [Eq. (5), 44]:

$$
\begin{aligned}
& p_{x}(x)= \\
& \int_{0}^{\infty} \int_{0}^{\infty} \frac{1}{x_{2} x_{3}} \frac{x}{x_{2} x_{3}} \frac{x_{2}}{\Omega_{2}} \frac{x_{3}}{\Omega_{3}} \frac{1}{\Omega_{1}} \mathrm{e}^{-\frac{1}{\Omega_{1}}\left(\frac{x}{x_{2} x_{3}}\right)^{2}-\frac{x_{2}^{2}}{\Omega_{2}}-\frac{x_{3}^{2}}{\Omega_{3}}} \mathrm{~d} x_{2} \mathrm{~d} x_{3}= \\
& \quad=\frac{8}{\Omega_{1} \Omega_{2} \Omega_{3}} x \int_{0}^{\infty} x_{2}^{-1} K_{0}\left(2 \sqrt{\frac{1}{\Omega_{1} \Omega_{3}} \frac{x^{2}}{x_{2}^{2}}}\right) \mathrm{d} x_{2}
\end{aligned}
$$


where $K_{n}(x)$ is the modified Bessel function of the second kind [Eq. (1.1), 47].

In Fig. 6 [Fig. 1, 44], some curves are presented showing PDF of the product of three Rayleigh RVs depending on that product for more values of the signal's average power $\Omega_{i}$ at all sections. One may notice that PDF increases at low $x$ values for all of Rayleigh signal powers values, then reaches its maximum and decreases at higher signal envelope values.

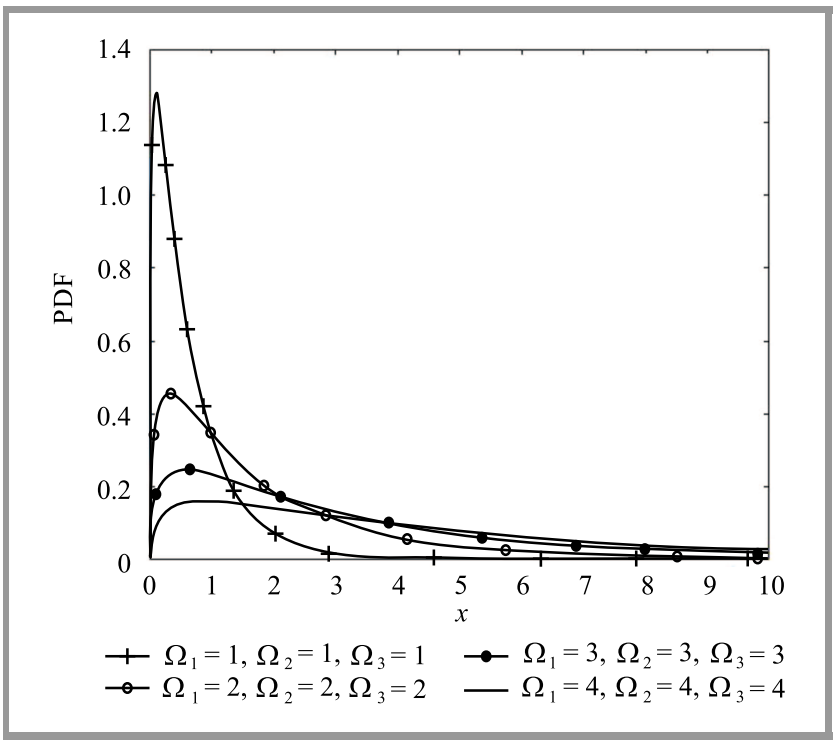

Fig. 6. PDF of the product of three Rayleigh RVs for different signal powers values.

We can notice from this figure that small amplitudes affect the PDF curves more distinctly.

\subsection{CDF of the Product of Three Rayleigh RVs}

The cumulative distribution function of $x$ (product of three Rayleigh RVs) was derived in [Eq. (6), 44] as:

$$
\begin{gathered}
F_{x}(x)=\int_{0}^{x} p_{x}(t) \mathrm{d} t=\frac{8}{\Omega_{1} \Omega_{2} \Omega_{3}} x^{2} \sum_{j_{1}=0}^{\infty} \frac{1}{2\left(j_{1}\right)} \frac{x^{2 j_{1}}}{\Omega_{1}^{j_{1}}} \\
\times \int_{0}^{\infty} \mathrm{d} x_{2} x_{2}^{-1} \mathrm{e}^{-\frac{1}{\Omega_{2}} x_{2}^{2}}\left(\frac{\Omega_{3}}{\Omega_{1}} x^{2}\right)^{-j_{1}} K_{-2 j_{1}}\left(2 \sqrt{\frac{x^{2}}{\Omega_{1} \Omega_{3} x_{2}^{2}}}\right) .
\end{gathered}
$$

\subsection{OP of the Product of Three Rayleigh RVs}

Outage probability is defined as the probability of the information rate (signal envelope, SNR or signal-to-interference ratio (SINR)) being lower than the information rate threshold value.

It is the probability of an outage occurring within a specified time period and determined by the following formula [Eq. (2.23), 47]:

$$
O P=P(X \leq x)=\int_{0}^{x} p_{x}(t) \mathrm{d} t=F_{x}(x) .
$$

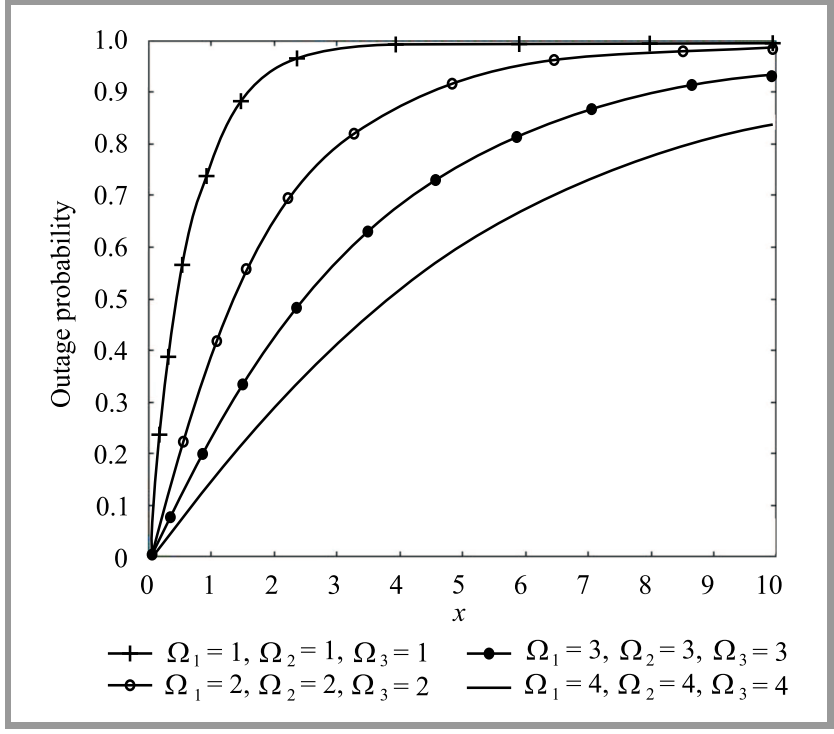

Fig. 7. OP of the product of three Rayleigh random variables for different average signal envelope powers $\Omega_{i}$.

It is mathematically the CDF from Eq. (4).

OP is presented in Fig. 7 [Fig. 2, 44] for a few values of the average signal envelopes' powers from each of the sections. It was concluded that OP grows along with an increase in signal envelope $x$ and achieves its maximum value. Likewise, it is evident that OP drops with the increase in $\Omega_{i}$.

\subsection{Moments of the Product of Three Rayleigh RVs}

Moment is a quantitative measure that describes the shape of a function in mathematics. It is used both in mechanics and statistics. If the function concerned is a probability distribution function, then moments have the following meanings:

- zero-th moment is the total probability,

- first moment is the mean of the signal, i.e. the expected value,

- second moment is the variance or the signal's average power,

- third moment is the skewness,

- fourth moment is the kurtosis.

For the product of three Rayleigh RVs, the first moment $m_{1}$, or the mean of the signal, is defined by:

$$
\begin{aligned}
m_{1} & =\bar{x}=\int_{0}^{\infty} x p_{x}(x) \mathrm{d} x= \\
& =\int_{0}^{\infty} x^{2} \frac{8}{\Omega_{1} \Omega_{2} \Omega_{3}} \int_{0}^{\infty} x_{2}^{-1} K_{0}\left(2 \sqrt{\frac{1}{\Omega_{1} \Omega_{3}} \frac{x^{2}}{x_{2}^{2}}}\right) \mathrm{d} x_{2} \mathrm{~d} x .
\end{aligned}
$$


The $n$-th moment of an independent Rayleigh distributed $\mathrm{RV}$ is given by Eq. (3) in [39]:

$$
E\left[x_{i}^{n}\right]=\Omega_{i}^{n}\left[\Gamma\left(\frac{n}{2}+1\right)\right] .
$$

If we use Eq. (8) derived in [48], and enter Nakagami- $m$ parameters as $m_{1}=m_{2}=m_{3}=1$, the first moment of the product of three Rayleigh RVs may be expressed as:

$$
m_{1}=\sqrt{\Omega_{1} \Omega_{2} \Omega_{3}}[\Gamma(3 / 2)]^{3} .
$$

The second moment, $m_{2}$, is:

$$
\begin{aligned}
& m_{2}=\overline{x^{2}}=\int_{0}^{\infty} x^{2} p_{x}(x) \mathrm{d} x= \\
& =\frac{8}{\Omega_{1} \Omega_{2} \Omega_{3}} \int_{0}^{\infty} x^{3} \int_{0}^{\infty} x_{2}^{-1} K_{0}\left(2 \sqrt{\frac{1}{\Omega_{1} \Omega_{3}} \frac{x^{2}}{x_{2}^{2}}}\right) \mathrm{d} x_{2} \mathrm{~d} x= \\
& \quad=\left(\Omega_{1} \Omega_{2} \Omega_{3}\right)[\Gamma(2)]^{3}
\end{aligned}
$$

The second moment is the variance. The standard deviation is a square root of the variance. In telecommunications, this is the average power of the signal.

The normalized third central moment is the skewness. In the probability theory and statistics, skewness is the size of the limitedness of distribution. It is a measure of symmetry or, more precisely, of a lack of symmetry. Distribution of a data set is symmetrical if it looks the same on the left- and right-hand side of the center value. In this case, the third moment is equal to zero. Also, skewness may assume positive or negative values, or even may be undefined.

A distribution that is skewed to the left, i.e. the tail of the distribution is longer on the left-hand side of the curve, has negative skewness and vice versa, a distribution skewed to the right has positive skewness. So, the third moment, $m_{3}$, or the skewness, is:

$$
\begin{aligned}
& m_{3}=\overline{x^{3}}=\int_{0}^{\infty} x^{3} p_{x}(x) \mathrm{d} x= \\
& =\frac{8}{\Omega_{1} \Omega_{2} \Omega_{3}} \int_{0}^{\infty} x^{4} \int_{0}^{\infty} x_{2}^{-1} K_{0}\left(2 \sqrt{\frac{1}{\Omega_{1} \Omega_{3}} \frac{x^{2}}{x_{2}^{2}}}\right) \mathrm{d} x_{2} \mathrm{~d} x
\end{aligned}
$$

As for previous moments, the final formula is obtained in a similar manner, from [Eq. (10), 48]:

$$
m_{3}=\left(\Omega_{1} \Omega_{2} \Omega_{3}\right)^{3 / 2}[\Gamma(5 / 2)]^{3} \text {. }
$$

In Fig. 8, the moments of the product of three Rayleigh RVs are shown. One may see from this figure that all moments decrease with increasing $\Omega$, in line with theoretical expectations.

The fourth moment, $m_{4}$, the kurtosis, is a measure of whether the data are sharper or flatter distributed relative to normal distribution. This means that a kurtosis dataset

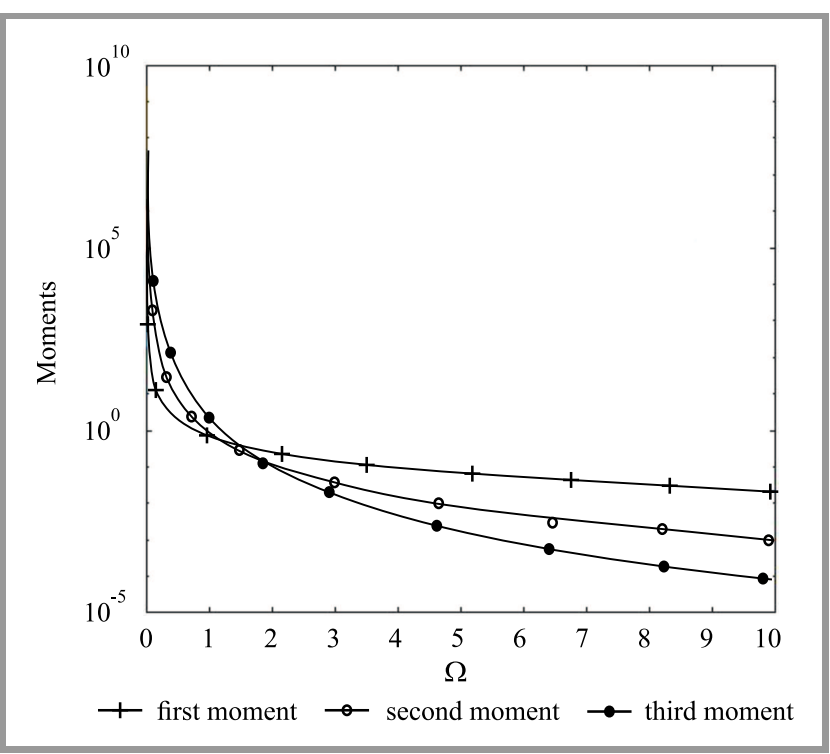

Fig. 8. Moments of the product of three Rayleigh RVs depending on signal power $\Omega=\Omega_{1}=\Omega_{2}=\Omega_{3}$.

has a large, significant peak near the mean value, suddenly decreasing and having long tails.

A dataset with a low kurtosis has a flat tip near the middle. Uniform distribution is an extreme case and standard normal distribution has a kurtosis equal to zero. In this case, positive kurtosis indicates distribution with a peak, and negative kurtosis indicates that distribution is more even. Finally, the moment of $n$-th order, $m_{n}$ is:

$$
\begin{aligned}
& m_{n}=\overline{x^{n}}=\int_{0}^{\infty} x^{n} p_{x}(x) \mathrm{d} x= \\
& =\frac{8}{\Omega_{1} \Omega_{2} \Omega_{3}} \int_{0}^{\infty} x^{x+1} \int_{0}^{\infty} x_{2}^{-1} K_{0}\left(2 \sqrt{\frac{1}{\Omega_{1} \Omega_{3}} \frac{x^{2}}{x_{2}^{2}}}\right) \mathrm{d} x_{2} \mathrm{~d} x= \\
& \quad=\left(\Omega_{1} \Omega_{2} \Omega_{3}\right)^{n / 2}[\Gamma(1+n / 2)]^{3}
\end{aligned}
$$

\subsection{AoF of the Product of Three Rayleigh RVs}

The severity of fading is defined by a measure known as the amount of fading [49]. This measure is used to describe different models which define the fading condition in radio channels. Here, we present comprehensive insights illustrating how faded a given channel is, based on fading severity in radio channels. This parameter does not depend on the mean power. It depends on the current signal value, SNR or SINR.

The amount of fading, connected with PDF of fading, is defined by the ratio of the variance of the received energy and the square of the mean of the received energy [Eq. (2), 49], [Eq. (2.5), 50]:

$$
\mathrm{AoF}=\frac{\operatorname{var}\left(x^{2}\right)}{\left(\mathrm{E}\left[x^{2}\right]\right)^{2}},
$$

with $\operatorname{var}($.$) denoting variance and E[$.$] denoting the statis-$ tical average value or the mathematical expectation. 
In $[49$, p. 29; 6], Charash gave this formula for AoF as a unified measure of the severity of the fading. AoF is usually independent of the average fading power.

Amount of fading is obtained by developing the formula below; so, it is specified by [Eq. (9), 51]:

$$
\begin{aligned}
\mathrm{AoF}=\frac{\operatorname{var}\left(x^{2}\right)}{\left(\mathrm{E}\left[x^{2}\right]\right)^{2}}=\frac{\mathrm{E}\left[\left(x^{2}-\Omega\right)^{2}\right]}{\Omega^{2}}= \\
=\frac{\mathrm{E}\left[x^{2}\right]-(\mathrm{E}[x])^{2}}{(\mathrm{E}[x])^{2}}=\frac{\mathrm{E}\left[x^{2}\right]}{(\mathrm{E}[x])^{2}}-1 .
\end{aligned}
$$

AoF may be expressed directly from the moments as:

$$
\mathrm{AoF}=\frac{m_{2}}{m_{1}^{2}}-1
$$

In Fig. 9, the amount of fading of the product of three Rayleigh RVs, i.e. of a three-hop Rayleigh channel, is presented versus average power envelopes $\Omega$. It is worth noting that AoF is independent of $\Omega$, which is in compliance with the theoretical assumptions that AoF typically does not depend on average power. As stated earlier, AoF provides a quantitative measure of fading that exists in wireless channels. Since the definition of AoF depends on two moments only (squared first and second moments of the power), it is possible to obtain the same value of AoF with more different PDFs.

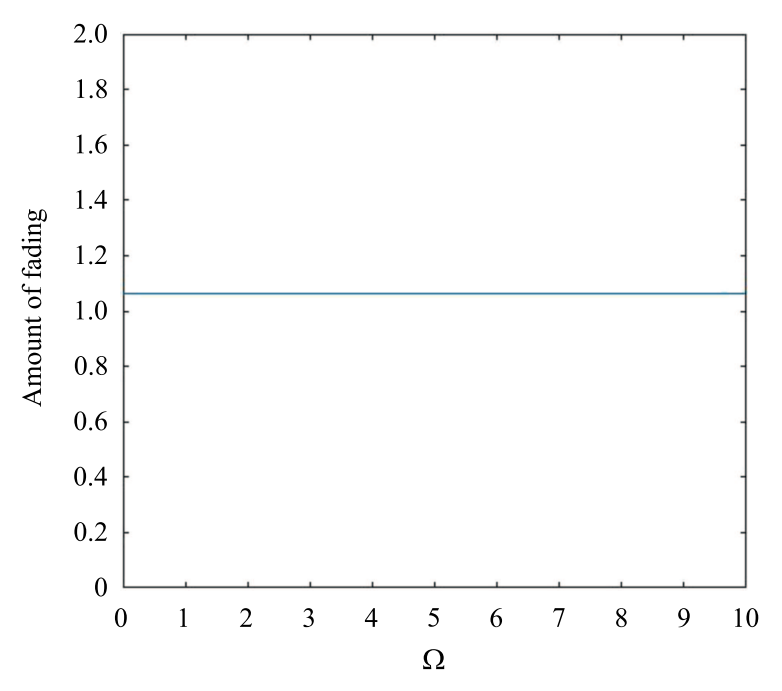

Fig. 9. Amount of fading of the product of three Rayleigh RVs versus signal power $\Omega$.

In other words, quantification of fading that is defined in Eq. (12) does not fully quantify the measured statistics of the fading channel, or PDF of the received power [51]. The reasons for the signal power going below a predefined threshold are: data rate, coding, modulation, demodulation. Because of that, outage probabilities are used as well for quantification of the performance of the fading channels, as presented in Subsection 2.3 for this scenario.

\section{Second Order Characteristics of the Product of Three Rayleigh Random Variables}

\subsection{LCR of the Product of Three Rayleigh RVs}

LCR of $X$ at threshold $x$ is defined as the rate at which the random process crosses a given level $x$ in the negative (or positive) direction [52]. To derive the LCR, we need to determine the joint probability density function (JPDF) between $x$ and $\dot{x}, p_{x \dot{x}} x \dot{x}$, and apply the Rice's formula [Eq. (2.106), 53]. So, LCR of the product $x$ of three Rayleigh RVs is:

$$
N_{X}(x)=\int_{0}^{\infty} \dot{x} p_{x \dot{x}}(x \dot{x}) \mathrm{d} \dot{x} .
$$

After adequate replacements, LCR of $x$ is [Eq. (15), 44]:

$$
\begin{aligned}
& N_{X}=\frac{1}{\sqrt{2 \pi}} \pi f_{m} \Omega_{1}^{\frac{1}{2}} x \frac{8}{\Omega_{1} \Omega_{2} \Omega_{3}} \times \\
& \int_{0}^{\infty} \int_{0}^{\infty} \mathrm{e}^{-\frac{1}{\Omega_{1}} \frac{x^{2}}{x_{2}^{2} x_{3}^{2}}-\frac{1}{\Omega_{2}} x_{2}^{2}-\frac{1}{\Omega_{3}} x_{3}^{2}} \sqrt{1+\frac{x^{2}}{x_{2}^{4} x_{3}^{2}} \frac{\Omega_{2}}{\Omega_{1}}+\frac{x^{2}}{x_{2}^{2} x_{3}^{4}} \frac{\Omega_{3}}{\Omega_{1}}} \mathrm{~d} x_{2} \mathrm{~d} x_{3} .
\end{aligned}
$$

It is solved in [44] by using Laplace approximation theorem [54] and presented here in Fig. 10 [Fig. 3, 44]. In this figure, LCR, normalized by $f_{m}$, is shown versus the signal envelopes for different signal powers $\Omega_{i}$. It is visible that LCR is increasing for small values of the signal envelope, reaches the maximum and then decreases for higher $x$ values. Small signal envelopes have a greater impact on LCR. LCR is higher for bigger $\Omega_{i}$. The system has better performance for lower LCR values.

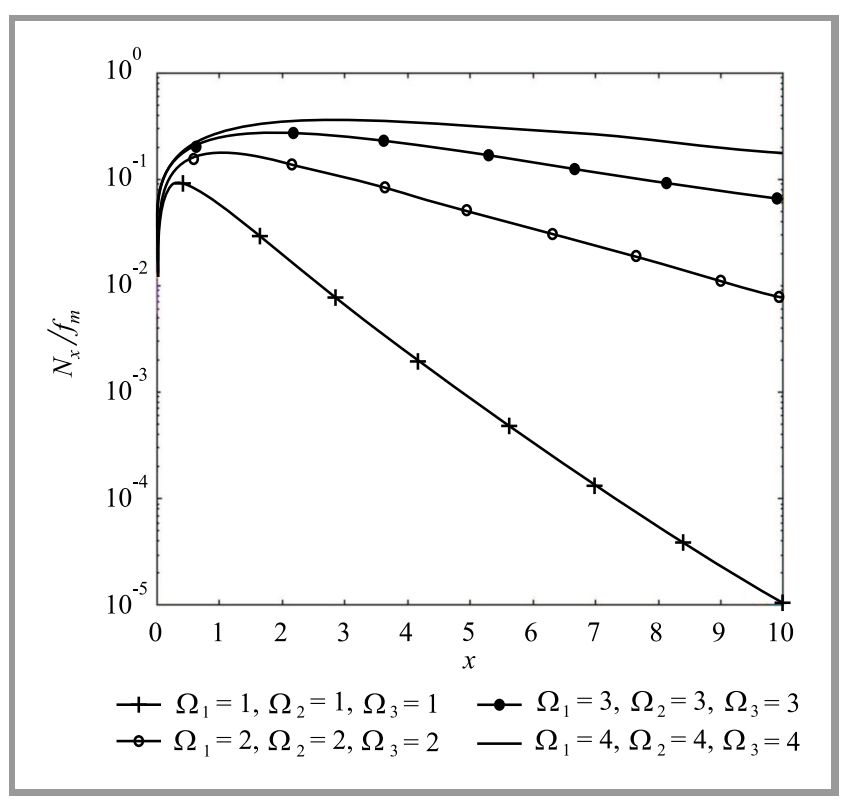

Fig. 10. LCR normalized by $f_{m}$ versus signal envelope $x$ for different values of signal power $\Omega_{i}$ 


\subsection{AFD of the Product of Three Rayleigh RVs}

AFD of RV at threshold $x$ defined as the average time over which the product of three Rayleigh RVs remains below $x$ after passing the defined level in the downward (or upward) direction. It is the ratio of the OP and the LCR given by [55]:

$$
T_{x}(x)=\frac{F_{x}(x)}{N_{x}(x)},
$$

with $F_{x}(x)$ from Eq. (4) denoting the CDF of $x$ evaluated at $X$. The LCR $N_{x}(x)$ is given in Eq. (14).

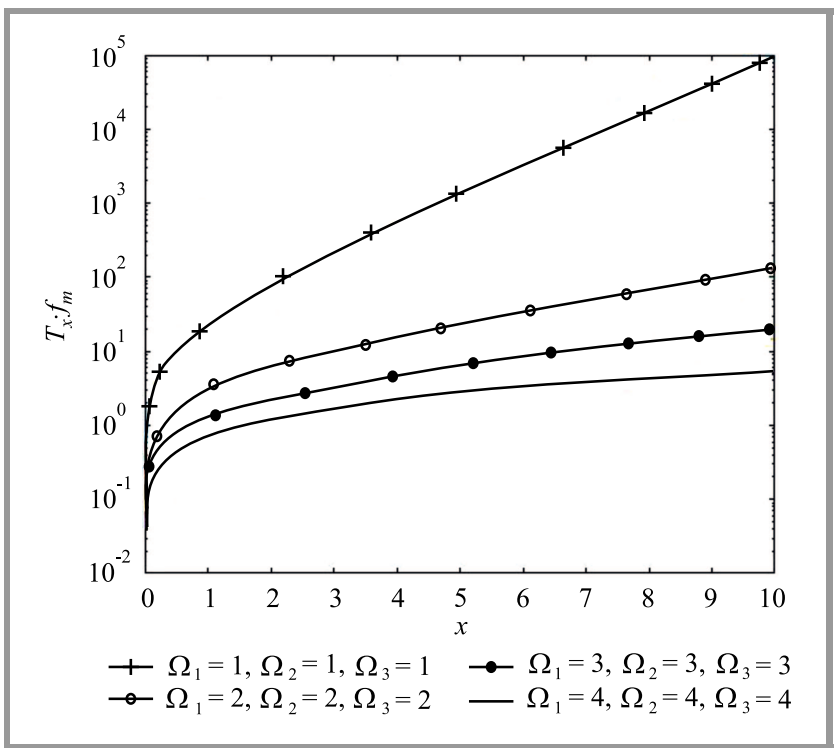

Fig. 11. AFD normalized by $f_{m}$ depending on the signal envelope for some values of signal powers $\Omega_{i}$.

In Fig. 11, [Fig. 4, 44], normalized AFD $\left(T_{x} f_{m}\right)$ is shown depending on signal envelope $x$. It is visible that AFD is increasing for all signal envelopes and also that AFD has smaller values for bigger power values.

\section{Conclusion}

Owing to transmit power limitations, multi-hop communication in relay systems offers a very contemporary approach to improving transmission in cellular and ad-hoc networks. The advantages of multi-hop relaying are particularly pronounced for rural areas with small populations and low traffic densities [56].

In this article, we presented formulas for calculating PDF and $\mathrm{CDF}$, and derived exact and simple closed-form formulas for moments of the product of three Rayleigh RVs. Based on these formulas, the outage probability and amount of fading for cascaded fading channels were worked out. LCR and AFD values for the same scenario, as obtained in [30], are also shown to complement the performance of a system of cascaded three-hop Rayleigh fading channels.
Our future work will be related to determining the performance for the product of multiple RVs with other general fading distributions $\kappa-\mu, \eta-\mu, \alpha-\mu, \alpha-\eta-\mu \ldots)$ used in multi-hop wireless relay communication systems.

\section{Acknowledgements}

This work is done under projects III-44006 and TR-33035 of the Serbian Ministry of Education, Science and Technological Development.

\section{References}

[1] Y. Chen, G. K. Karagiannidis, H. Lu, and N. Cao, "Novel approximations to the statistics of products of independent random variables and their applications in wireless communications", IEEE Trans. on Veh. Technol., vol. 61, no. 2, pp. 443-454, 2012 (DOI: 10.1109/TVT.2011.2178441).

[2] S. Nadarajaha and D. K. Dey, "On the product and ratio of $t$ random variables", Appl. Methem. Lett., vol. 19, no. 1, pp. 45-55, 2006 (DOI: 10.1016/j.aml.2005.01.004).

[3] N. Zlatanov, Z. Hadzi-Velkov, and G. K. Karagiannidis, "Level crossing rate and average fade duration of the double Nakagami- $m$ random process and application in MIMO keyhole fading channels", IEEE Commun. Lett., vol. 12, no. 11, pp. 822-824, 2008 (DOI: 10.1109/LCOMM.2008.081058).

[4] K. Pearson, "The problem of the random walk", Nature, vol. 72, p. 294, 1905 (DOI: 10.1038/072294b0).

[5] J. D. Donahue, "Products and quotients of random variables and their applications", ARL 64-115 Report, The Martin Company, Denver, Colorado, July 1964 [Online]. Available: https://apps.dtic.mil/ dtic/tr/fulltext/u2/603667.pdf

[6] M. D. Springer and W. E. Thompson, "The distribution of products of independent random variables", SIAM J. on Appl. Mathem., vol. 14, pp. 511-526, 1966 (DOI: 10.1137/0114046).

[7] M. D. Springer and W. E. Thompson, "The distribution of products of beta, gamma and Gaussian random variables", SIAM J. on Appl. Mathem., vol. 18, pp. 721-737, 1970 (DOI: 10.1137/0118065).

[8] Z. A. Lomnicki, "On the distribution of products of random variables", J. of the Royal Statist. Soc. Series B (Methodological), vol. 29, no. 3, pp. 513-524, 1967 (DOI: 10.1016/j.crma.2015.10.019)

[9] A. G. Glen, L. M. Leemis, and J. H. Drew, "Computing the distribution of the product of two continuous random variables", Computat. Statist. and Data Anal., vol. 44, pp. 451-464, 2004 (DOI: 10.1016/S0167-9473(02)00234-7).

[10] T. S. Glickman and F. Xu, "The distribution of the product of two triangular random variables", Statist. \& Probab. Lett., vol. 78, no. 16, pp. 2821-2826, 2008 (DOI: 10.1016/j.spl.2008.03.031).

[11] G. K. Karagiannidis, N. C. Sagias, and P. T. Mathiopoulos, "N*Nakagami: a novel stochastic model for cascaded fading channels", IEEE Trans. Commun., vol. 55, no. 8, pp. 1453-1458, 2007 (DOI: 10.1109/TCOMM.2007.902497).

[12] Z. Zheng, L. Wei, J. Hamalainen, and O. Tirkkonen, "Approximation to distribution of product of random variables using orthogonal polynomials for lognormal density", IEEE Trans. Commun., vol. 16, no. 12 , pp. 2028-2031, 2012 (DOI: 10.1109/LCOMM.2012.101712.122141).

[13] E. J. Leonardo and M. D. Yacoub, "Statistics of the product of arbitrary $\alpha-\mu$ variates with applications", in in Proc. 25th Int. Symp. on Pers., Indoor and Mob. Radio Commun. PIMRC 2014, Washington, USA, 2014, pp. 73-76 (DOI: 10.1109/PIMRC.2014.7136135). 
[14] Z. Stojanac, D. Suess, and M. Kliesch, "On products of Gaussian random variables", arXiv:1711.10516 [math.PR], 2018.

[15] N. Bhargav et al., "On the product of two $\kappa-\mu$ random variables and its application to double and composite fading channels", IEEE Trans. on Wirel. Commun., vol. 17, pp. 2457-2470, 20182018 (DOI: 10.1109/TWC.2018.2796562).

[16] D. H. Pavlovic et al., "Statistics for ratios of Rayleigh, Rician, Nakagami-m, and Weibull distributed random variables", Mathem. Problems in Engin., vol. 2013, Article ID 252804, 2013 (DOI: 10.1155/2013/252804).

[17] S. Ahmed, L. L. Yang, and L. L. Hanzo, "Probability distributions of products of Rayleigh and Nakagami- $m$ variables using Mellin transform", in Proc. IEEE Intern. Conf. on Commun. ICC 2011, Kyoto, Japan, 2011 (DOI: 10.1109/icc.2011.5963344).

[18] T. Taniguchi, Y. Karasawa, and M. Tsuruta, "An analysis method of double fading MIMO channels including LOS environments", in Proc. IEEE 19th Int. Symp. on Pers., Indoor and Mob. Radio Commun., Cannes, France, 2008 (DOI:10.1109/pimrc.2008.4699512).

[19] D. Krstic, M. Stefanovic, R. Gerov, and Z. Popovic, "Wireless relay system with two sections in $\kappa-\mu$ short term fading channel", in Proc. 12th Int. Conf. on Wirel. and Mob. Commun. ICWMC 2016, Barcelona, Spain, 2016, pp. 110-114 [Online]. Available: https://www.academia.edu/29873047/ICWMC_-_2016_The_Twelfth_ International_Conference_on_Wireless_and_Mobile_Communications

[20] N. Bhargav et al., "On the product of two $\kappa-\mu$ random variables and its application to double and composite fading channels", IEEE Trans. on Wirel. Commun., vol. 17, no. 4, pp. 2457-2470, 2018 (DOI:10.1109/twc.2018.2796562).

[21] A. M. Magableh, T. Aldalgamouni, and N. M. Jafreh, "Capacity analysis of dual-hop wireless communication systems over $\kappa-\mu$ fading channels", Comp. and Elec. Engin., vol. 40, no. 2, pp. 399-406, 2014 (DOI: 10.1016/j.compeleceng.2013.04.018).

[22] T. A. Tsiftsis, G. K. Karagiannidis, and S. A. Kotsopoulos, "Dualhop wireless communications with combined gain relays", IEE Proceedings - Communications, vol. 152, no. 5, pp. 528-532, 2005 (DOI: 10.1049/ip-com:200452685).

[23] M. Delibasic and M. Pejanovic-Djurisic, "Dual-hop amplify-andforward relay system over non-identical Ricean fading channels", Wirel. Pers. Commun., vol. 93, pp. 675-686, 2014 (DOI: 10.1007/s11277-014-2221-1).

[24] A. M. Magableh, T. Aldalgamouni, S. Mater, and O. Badarneh, "Ergodic capacity and SNR analysis for dual hop amplify and forward cooperative communication systems over $\alpha-\eta-\mu$ channels", IEEE Trans. on Veh. Technol., vol. 67, no. 12, pp. 12384-12388, 2018 (DOI: 10.1109/tvt.2018.2873485)

[25] S. Panić, S. Mohanchenko, C. Stefanović, and M. Stefanović, "First order outage statistics of asymmetrical RF-OW dual-hop relay communications", University thought, Publication in Natural Sciences, vol. 10, 2020 (DOI: 10.5937/univtho10-23091).

[26] E. Zedini, I. S. Ansari, and M. Alouini, "Performance analysis of mixed Nakagami- $m$ and gamma-gamma dual-hop FSO transmission systems", IEEE Photonics J., vol. 7, no. 1, pp. 1-20, 2015 (DOI: 10.1109/jphot.2014.2381657).

[27] N. I. Miridakis, D. D. Vergados, and A. Michalas, "Dual-hop communication over a satellite relay and shadowed Rician channels", IEEE Trans. on Veh. Technol., vol. 64, no. 9, pp. 4031-4040, 2015 (DOI: 10.1109/tvt.2014.2361832).

[28] Z. Bhutto and W. Yoon, "Dual-hop cooperative relaying with beamforming under adaptive transmission in $\kappa-\mu$ shadowed fading environments", Electronics, vol. 8, no. 6, pp. 1-18, 2019 (DOI: 10.3390/electronics8060658).

[29] H. Shen, Z. Li, and C. Qiu, "A distributed three-hop routing protocol to increase the capacity of hybrid wireless networks", IEEE Trans. on Mob. Comput., vol. 14, no. 10, pp. 1975-1991, 2015 (DOI: $10.1109 / \mathrm{tmc} .2015 .2388476)$.

[30] U. Bendre Shital and R. P. Jadhav, "A survey on packet retrieval for three-hop routing protocol in hybrid wireless networks", Int. J. of Sci. and Res., vol. 4, pp. 2165-2168, 2013 [Online]. Available: https://www.ijsr.net/archive/v4i10/NOV151026.pdf
[31] D.-H. Kim, Y.-C. Ko, and S. Park, "Three-hop MIMO relaying systems in Gaussian broadcast channels", in Proc. 2nd Int. Conf. on Sig. Process. and Commun. Sys., Gold Coast, QLD, Australia, 2008 (DOI: 10.1109/ICSPCS.2008.4813661).

[32] D. Krstić et al., "Product of three random variables and its application in relay telecommunication systems in the presence of multipath fading", J. of Telecomm. and Informat. Technol. (JTIT), no. 1, pp. 83-92, 2019 (DOI: 10.26636/jtit.2019.130018).

[33] D. Krstic, M. Stefanovic, and P. Nikolić, "Level crossing rate of product of Nakagami- $m$ random variable, Rician random variable and Rayleigh random variable", in IEICE Inform. and Commun. Technol. Forum, Graz, Austria, 2018.

[34] D. Aleksić, D. Krstic, Z. Popovic, I. Dinić, and M. Stefanović, "Outage probability of wireless relay communication system with three sections in the presence of Nakagami- $m$ short term fading", Int. J. of Commun., vol. 1, pp. 199-204, 2016 [Online]. Available: https://www.iaras.org/iaras/filedownloads/ijoc/2016/005-0031.pdf

[35] D. Krstic, M. Stefanovic, P. Nikolić, and S. Minic, "Statistics of the product of three Nakagami- $m$ random variables with applications", in Proc. 26th Int. Conf. on Software, Telecommun. and Com. Netw. SoftCOM 22018, Split, Croatia, 2018, pp. 36-40 (DOI: 10.23919/SOFTCOM.2018.8555819).

[36] D. Krstic, M. Stefanovic, M. M. Bani Yassein, S. Aljawarneh, and P. Nikolic, "Statistics of the product of three Rician random processes with application", in Proc. of the 1st Int. Conf. on Data Science, E-learn. and Inform. Syst. DATA 2018, Madrit, Spain, 2018 (DOI: 10.1145/3279996.3280015).

[37] M. Hajiaghayi, M. Dong, and B. Liang, "Jointly optimal channel pairing and power allocation for multichannel multihop relaying", IEEE Trans. on Sig. Process., vol. 59, no. 10, pp. 4998-5012, 2011 (DOI: 10.1109/TSP.2011.2161475).

[38] I-H. Lee and D. Kim, "Outage probability of multi-hop MIMO relaying with transmit antenna selection and ideal relay gain over Rayleigh fading channels", IEEE Trans. on Commun., vol. 57, no. 2, pp. 357-360, 2009 (DOI: 10.1109/tcomm.2009.02.070058).

[39] J. Salo, H. M. El-Sallabi, and P. Vainikainen, "The distribution of the product of independent Rayleigh random variables", IEEE Intern. Conf. on Commun., vol. 54, no. 2, pp. 639-643, 2006 (DOI: 10.1109/TAP.2005.863087).

[40] Z. Hadzi-Velkov, N. Zlatanov, and G. K. Karagiannidis, "Level crossing rate and average fade duration of the multihop Rayleigh fading channel", in Proc. IEEE Int. Conf. on Commun. ICC 2008, Beijing, China, 2008 (DOI: 10.1109/ICC.2008.835).

[41] G. K. Karagiannidis, S. Nikos, and M. Takis, "N*Nakagami: a novel stochastic model for cascaded fading channels", IEEE Trans. on Commun., vol. 55, no. 8, pp. 1453-1458, 2007 (DOI: 10.1109/TCOMM.2007.902497).

[42] C. B. Issaid and M. S. Alouini, "Level crossing rate and average outage duration of free space optical links", IEEE Trans. on Commun., vol. 67, no. 9, pp. 6234-6242, 2019 (DOI: 10.1109/tcomm.2019.2918324).

[43] G. L. Stüber, Principles of Mobile Communication, 2nd ed. Kluwer Academic Publ., 2001 (ISBN: 9780792379980).

[44] D. Krstic, P. Nikolic, and M. Stefanovic, "The performance of threehop wireless relay channel in the presence of Rayleigh fading", in Image Processing and Communications, M. Choraś and R. S. Choraś, Eds. Adv. in Intell. Syst. and Comput. serie, vol. 1062, pp. 222-230. Springer, 2019 (ISBN: 9783030312534).

[45] P. M. Shankar, "Statistical models for fading and shadowed fading channels in wireless systems: A pedagogical perspective", Wireless Personal Communications, vol. 60, pp. 191-213, 2011 (DOI: 10.1007/s11277-010-9938-2).

[46] P. M. Shankar, "Fading and shadowing in wireless systems", New York, Dordrecht, Heidelberg, London: Springer, 2012 (ISBN: 9781461403678).

[47] Z. H. Yang and Y. M. Chu, "On approximating the modified Bessel function of the second kind", J. of Inequalit. and Appl., vol. 41, 2017 (DOI: 10.1186/s13660-017-1317-z). 
[48] D. B. Cheikh, "Outage probability formulas for cellular networks: contributions for MIMO, CoMP and time reversal features", Telecom Paris Tech, 2012, English [Online]. Available: https://pastel.archives-ouvertes.fr/pastel-00790614

[49] D. S. Krstic, P. B. Nikolic, I. Vulic, S. Minic, and M. C. Stefanovic, "Performance of the product of three Nakagami-m random variables", applied for publication in J. of Commun. Software and Systems (JCOMSS).

[50] U. Charash, "Reception through Nakagami fading multipath channels with random delays", IEEE Trans. on Commun., vol. 27, no. 4, pp. 657-670, 1979 (DOI: 10.1109/TCOM.1979.1094444).

[51] M. K. Simon and M. S. Alouini, Digital Communication over Fading Channels: A Unified Approach to Performance Analysis. New York: Wiley, 2000 (ISBN: 9780471317791).

[52] R. Priyadarshi, M. P. Singh, A. Bhardwaj, and P. Sharma, "Amount of fading analysis for composite fading channel using Holtzman approximation", in Proc. 4th Int. Conf. on Image Inform. Process., Himachal Pradesh, India, 2017 (DOI: 10.1109/iciip.2017.8313759).

[53] W. C. Jakes, Microwave Mobile Communications. Piscataway, NJ: IEEE Press, 1994 (ISBN: 978-078031069).

[54] S. O. Rice, "Mathematical analysis of random noise", Bell Syst. Tech. J., vol. 23,no. 3, pp. 282-332, 1944 (DOI: 10.1002/j.1538-7305.1944.tb00874.x).

[55] J. L. Lopez and P. J. Pagola, "A simplification of the Laplace method for double integrals. Application to the second Appell function", Electr. Trans. on Numer. Anal., vol. 30, pp. 224-236, 2008 [Online]. Available: https://www.emis.de//journals/ETNA/vol.30.23008/ pp224-236.dir/pp224-236.pdf

[56] I. K. Eltahir, K. H. Bilal, and S. Taha, "Evaluate comparative of cooperative relaying protocols in wireless communication", Int. J. of Scient. and Engin. Res., vol. 4, no. 8, pp. 112-116, 2013 [Online]. Available: https://www.ijser.org/onlineResearchPaperViewer.aspx? Evaluate-Comparative-of-Cooperative-Relaying-Protocols-inWireless-Communication.pdf

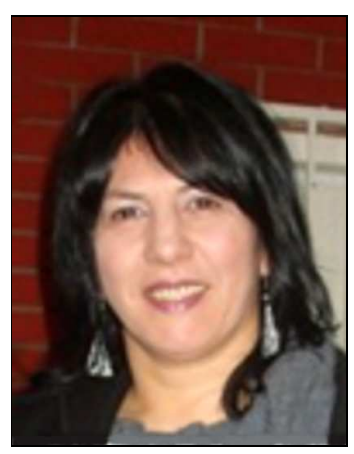

Dragana S. Krstic received her B.Sc., M.Sc. and Ph.D. degrees in Electrical Engineering from the Faculty of Electronic Engineering, University of Nis, Serbia, in 1990, 1998 and 2006, respectively. She has been working at the Faculty of Electronic Engineering, University of Nis, since 1990. Her research interests focus on the telecommunications theory, optical communication systems, wireless, mobile and satellite communication systems. She is the author and co-author, of approximately 260 scientific research papers, with 60 of them printed in international journals, several in national journals, and close to 140 presented at international symposia and conferences.

(iD) https://orcid.org/0000-0002-2579-3911

E-mail: dragana.krstic@elfak.ni.ac.rs

Faculty of Electronic Engineering

University of Nis

Aleksandra Medvedeva 14

18000 Nis, Serbia

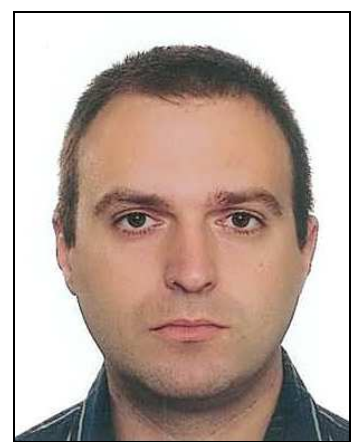

Petar B. Nikolic graduated from the Faculty of Electronic Engineering, University of Nis, Serbia, and received his M.Sc. and Ph.D. degrees in 2008 and 2016, respectively. He is currently working for Tigar Tyres, Pirot. His research interests focus on wireless communication systems. He has written or coauthored a considerable number of papers published in renowned journals and conference proceedings.

E-mail: nikpetar@gmail.com

TigarTyres

Pirot, Serbia

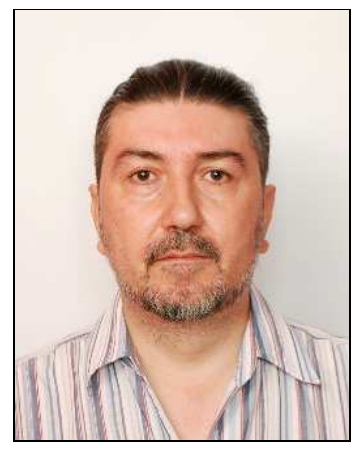

Zoran J. Popovic graduated the Faculty of Electronic Engineering, University of $\mathrm{Nis}$, Serbia, and received his M.Sc. and Ph.D. degrees in 2003 and 2011, respectively. His interests are in the field of telecommunications theory, as well as mobile and wireless communication systems. Currently, he is working as Professor at the Department of Information and Communications Technologies of the Higher Technical Professional School in Zvecan.

E-mail: z.popovix@live.com

Higher Technical School of Professional Studies Zvečan, Serbia

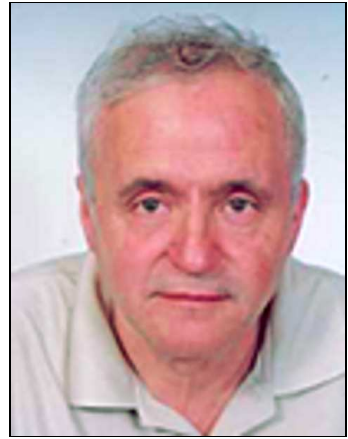

Mihajlo C. Stefanović received his B.Sc., M.Sc. and Ph.D. degrees in Electrical Engineering from the Faculty of Electronic Engineering, Department of Telecommunications, University of Nis, Serbia, in 1971, 1976 and 1979, respectively. Currently he is a retired professor at the Faculty of Electronic Engineering in Nis.

https://orcid.org/0000-0002-8063-8981

E-mail: mihajlo.stefanovic@elfak.ni.ac.rs

Faculty of Electronic Engineering

University of Nis

Aleksandra Medvedeva 14

18000 Nis, Serbia 